\title{
Continuous Fabrication of Slippery Liquid-Infused Coatings on Rolls of Flexible Materials
}

Harshit Agarwal, ${ }^{1, \dagger}$ William M. Breining, ${ }^{2, \dagger}$ and David M. Lynn ${ }^{1,2, *}$

${ }^{1}$ Department of Chemical \& Biological Engineering, University of Wisconsin-Madison, 1415 Engineering Dr., Madison, WI 53706, USA; ${ }^{2}$ Department of Chemistry, University of WisconsinMadison, 1101 University Avenue, Madison, WI 53706, USA.; ${ }^{\dagger}$ Equally contributing author; EMail: (D.M.L)dlynn@engr.wisc.edu

\section{Supporting Information}
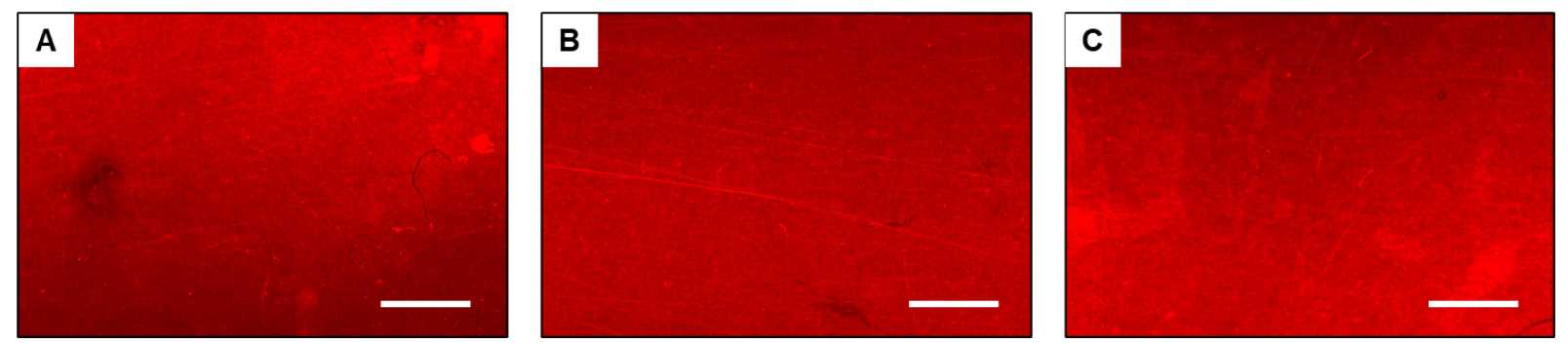

Figure S1. (A-C) Fluorescence microscope images of three representative sections (ends $(A, C)$ and middle (B) of a native (azlactone-containing) simultaneously sprayed PVDMA/PEI film after treatment with tetramethylrhodamine cadaverine. Scale bars $=400 \mu \mathrm{m}$. Panel B is reproduced from Figure $2 \mathrm{~B}$.

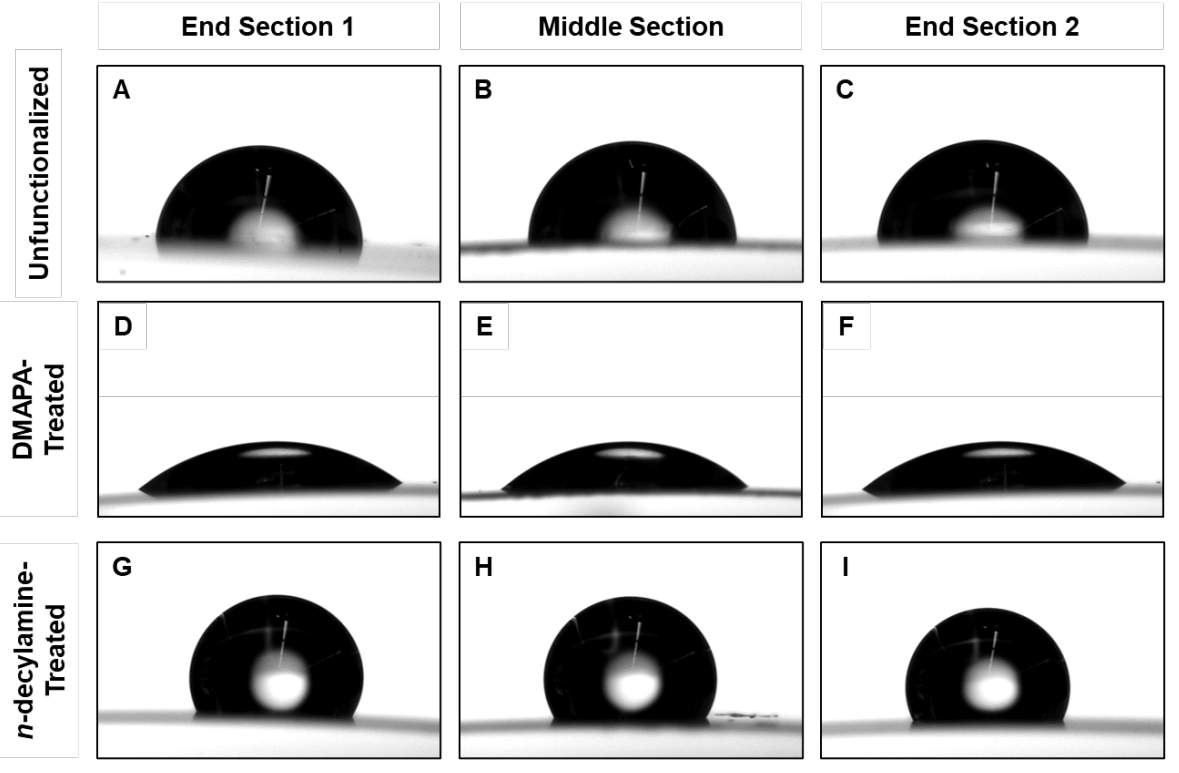

Figure S2. Images showing contact angles of $5 \mu \mathrm{L}$ water droplets on three sections (ends and middle (reproduced from Figure 2)) of simultaneously sprayed PVDMA/PEI films fabricated on a $30 \mathrm{~cm}$ long flexible polyester Dur-Lar film (A-C) before functionalization $\left(\theta \sim 86^{\circ}\right)$ and after functionalization with (D-F) DMAPA $\left(\theta \sim 42^{\circ}\right)$ and $(\mathrm{G}-\mathrm{I}) n$-decylamine $\left(\theta \sim 115^{\circ}\right)$ overnight at room temperature. Panels $\mathrm{B}, \mathrm{E}$, and $\mathrm{F}$ are reproduced from Figures $2 \mathrm{D}, \mathrm{E}$, and $\mathrm{F}$, respectively. 

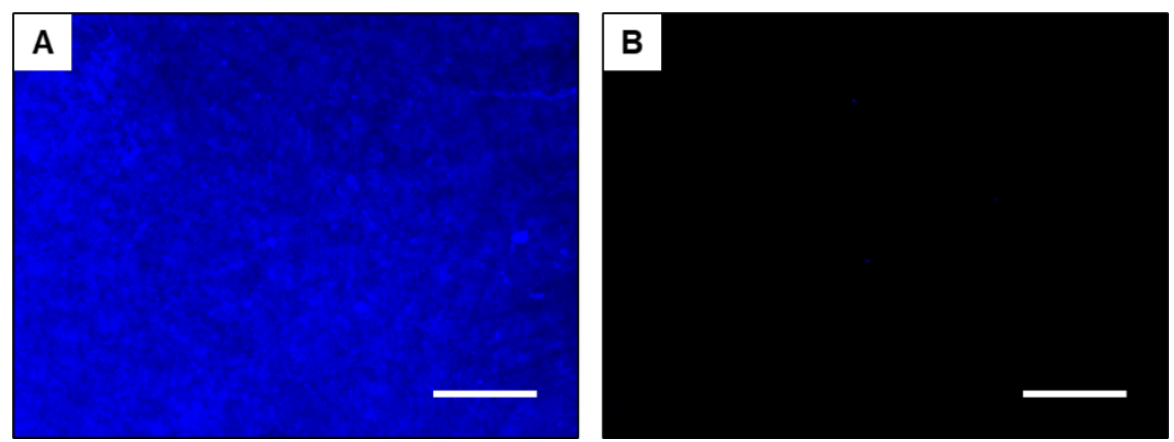

Figure S3. Fluorescence microscopy images of an (A) amine-containing (B) decanoyl chloridetreated PVDMA/PEI film after treatment with 7-dimethylaminocoumarin-4-acetic acid succinimidyl ester. Scale bar $=400 \mu \mathrm{m}$. The presence of fluorescence (false-colored blue) throughout the film in (A) (and the absence of fluorescence in (B)) confirms the presence of residual amine-based chemical functionality in the PVDMA/PEI film that is available for subsequent reaction with amine-reactive molecules.
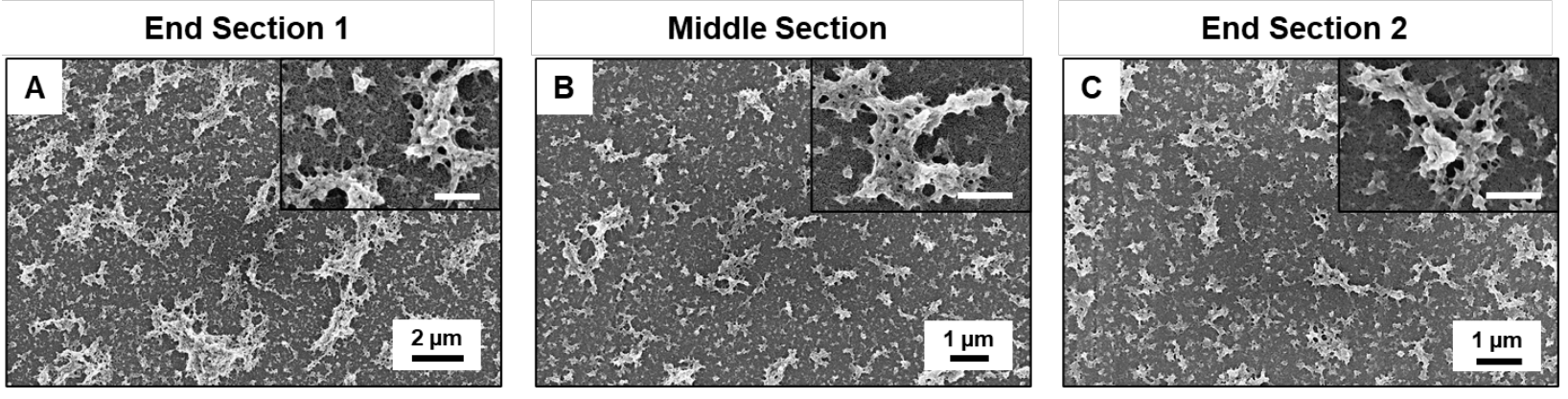

Figure S4. Low- and high-magnification (insets) SEM images ('top-down') of three sections (ends $(\mathrm{A}, \mathrm{C})$ and middle (B, reproduced from Figure 2 ) of simultaneously sprayed PVDMA/PEI films fabricated on a $30 \mathrm{~cm}$ long flexible polyester film (Dur-Lar)). Scale bar of insets $=400$ $\mathrm{nm}$. Panel B is reproduced from Figure $2 \mathrm{C}$. 


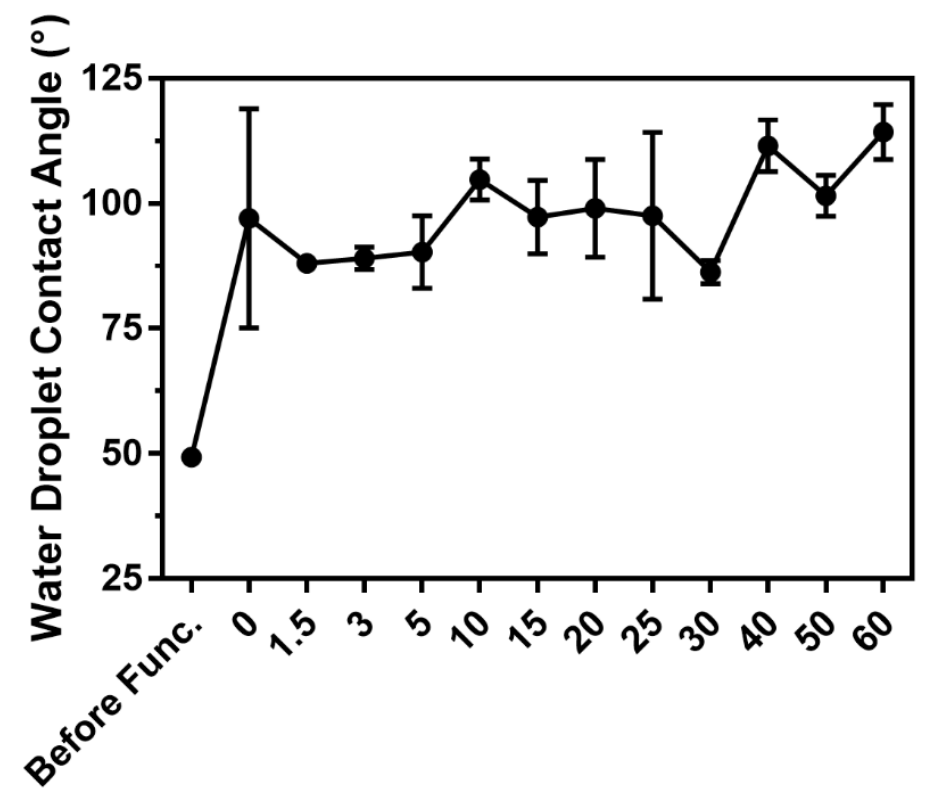

Time After Functionalization (min)

Figure S5. Plot showing the contact angle of $10 \mu \mathrm{L}$ water droplets on simultaneously sprayed PVDMA/PEI films fabricated on a $30 \mathrm{~cm}$ long flexible polyester film (Dur-Lar) both before and after treatment of the film by spraying a solution of $40 \mu \mathrm{L}$ of $n$-decylamine in $5 \mathrm{~mL}$ of DMF onto the film surface. Pieces of the film were cut, rinsed with acetone, and then characterized. 


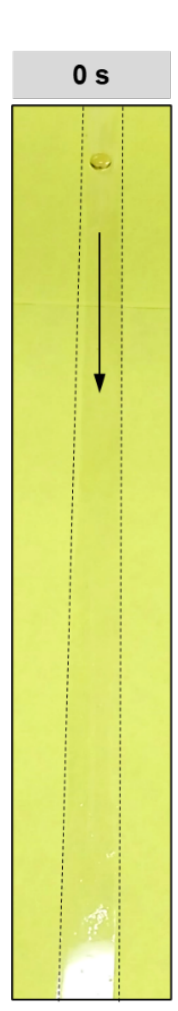

Beer

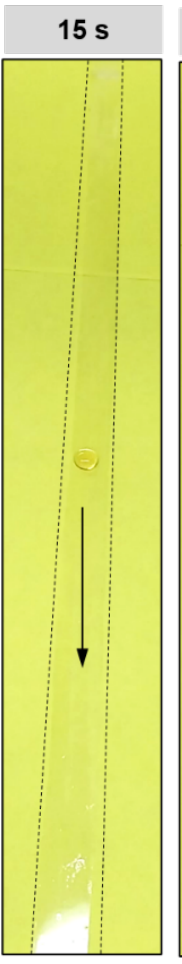

Tea

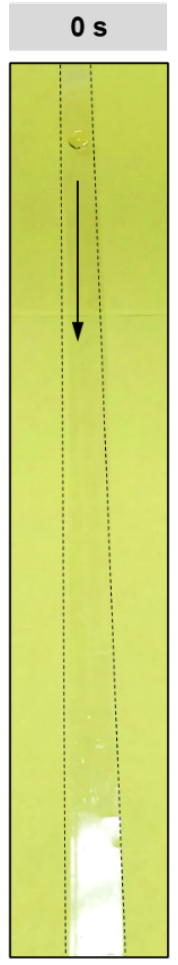

$10 \mathrm{~s}$

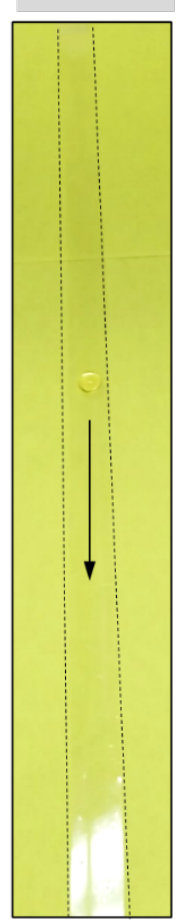

$20 \mathrm{~s}$

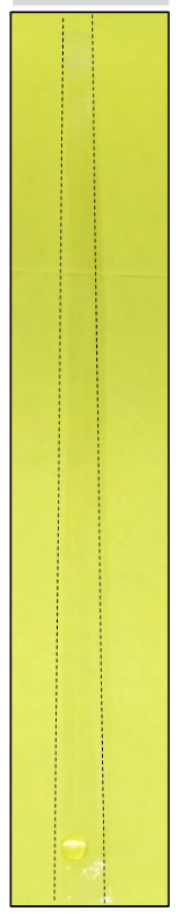

Lake Water

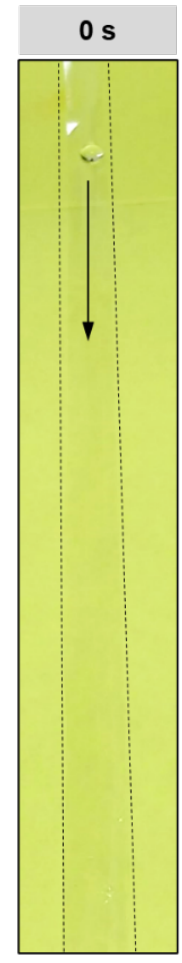

$20 \mathrm{~s}$

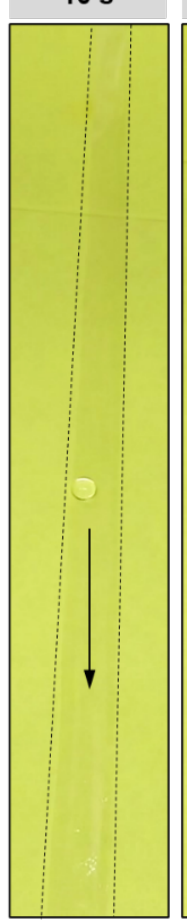

$20 \mathrm{~s}$

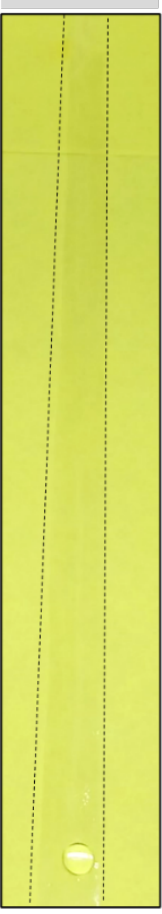

Figure S6. Angled top-down images showing droplets of various chemically complex fluids sliding on silicone oil-infused, simultaneously sprayed PVDMA/PEI films fabricated on a $30 \mathrm{~cm}$ long flexible polyester film (Dur-Lar). The films were treated with $n$-decylamine followed by treatment with $n$-decanoyl chloride before infusion with silicone oil. (Droplet volume $=100 \mu \mathrm{L}$; tilt angle $\approx 10^{\circ}$ ) 

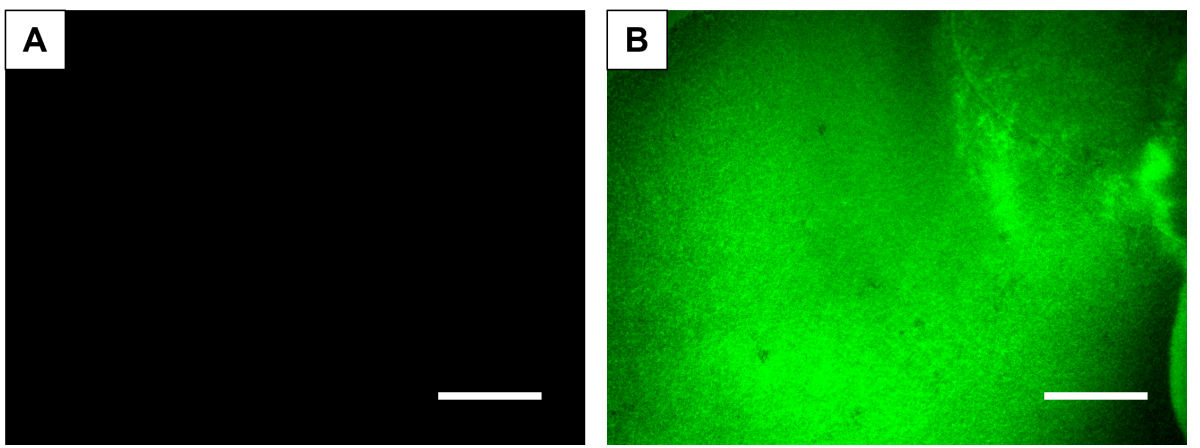

Figure S7. Fluorescence microscopy images of SLIPS-coated (A) and uncoated (B) PET films after incubation with Staphylococcus aureus for 24 hours; samples were treated with the fluorescent stain SYTO-9 prior to imaging (see Methods). Scale bars $=400 \mu \mathrm{m}$. The fluorescence (false-colored green) observed on the uncoated substrate in (B) indicates fouling by bacteria; the SLIPS-coated substrate shown in (A) remained free of bacterial fouling.
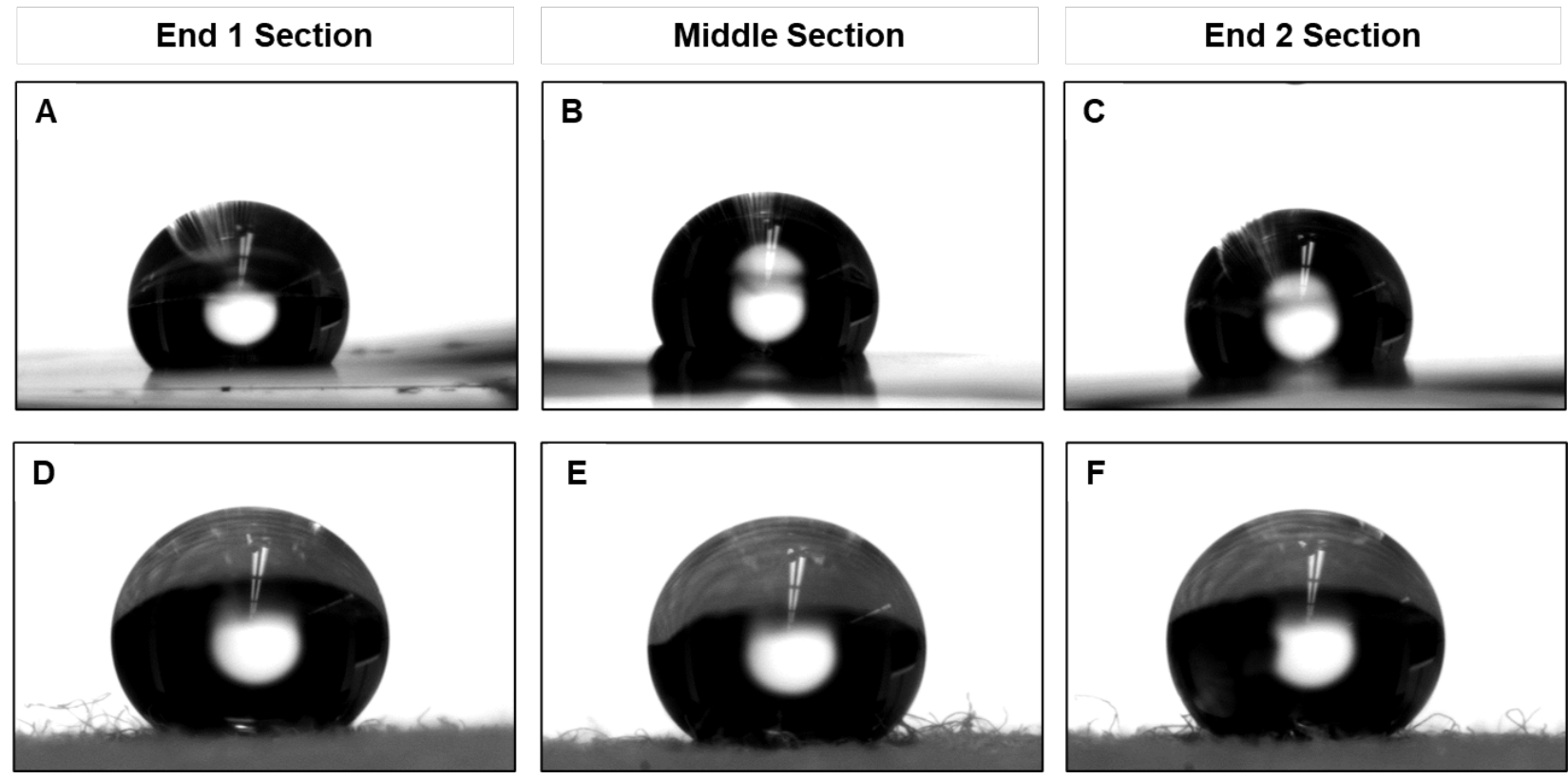

Figure S8. Images showing contact angles of water droplets on three sections (ends and middle) of simultaneously sprayed PVDMA/PEI films functionalized, post-fabrication, with decylamine and decanoyl chloride, fabricated on a $30 \mathrm{~cm}$ long strip of aluminum foil (A-C, $\theta \sim 110^{\circ}$ ) and woven cloth mesh (D-F, $\theta \sim 140^{\circ}$ ). Water droplet size $=5 \mu \mathrm{L}$ in the case of aluminum foil and 10 $\mu \mathrm{L}$ in the case of woven cloth mesh. 

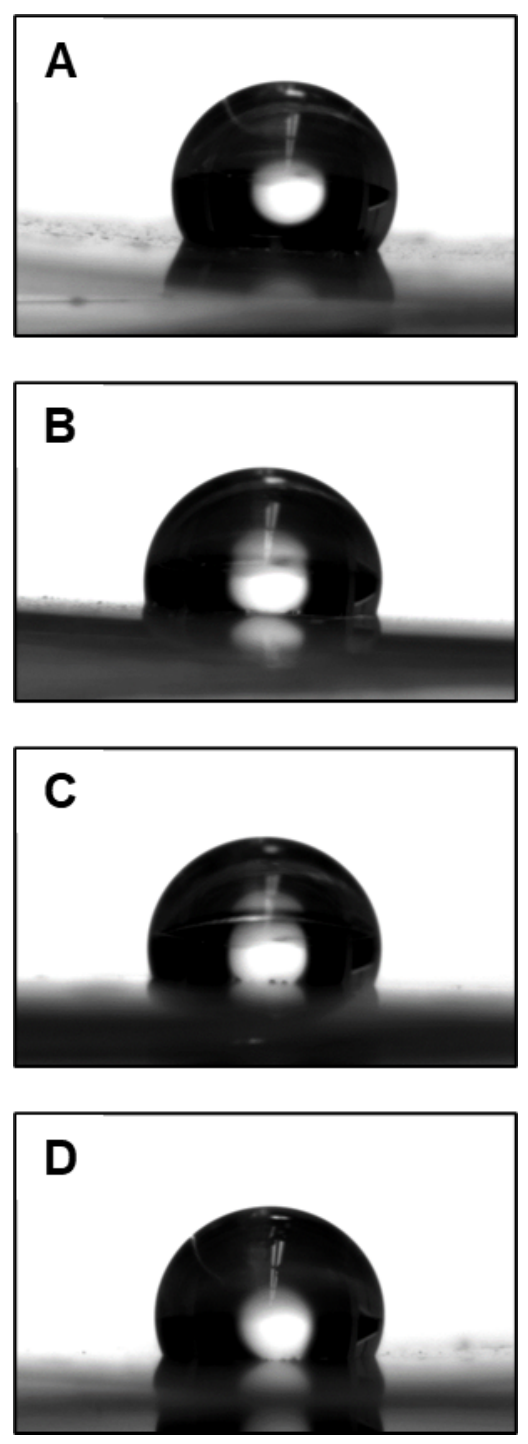

Figure S9. Images showing contact angles of $5 \mu \mathrm{L}$ water droplets at different points on a $150 \mathrm{~cm}$ long film coated using a roll-to-roll process. The film was functionalized with $n$-decylamine and decanoyl chloride after fabrication. Panel $\mathrm{C}$ is reproduced from Figure 6B. 

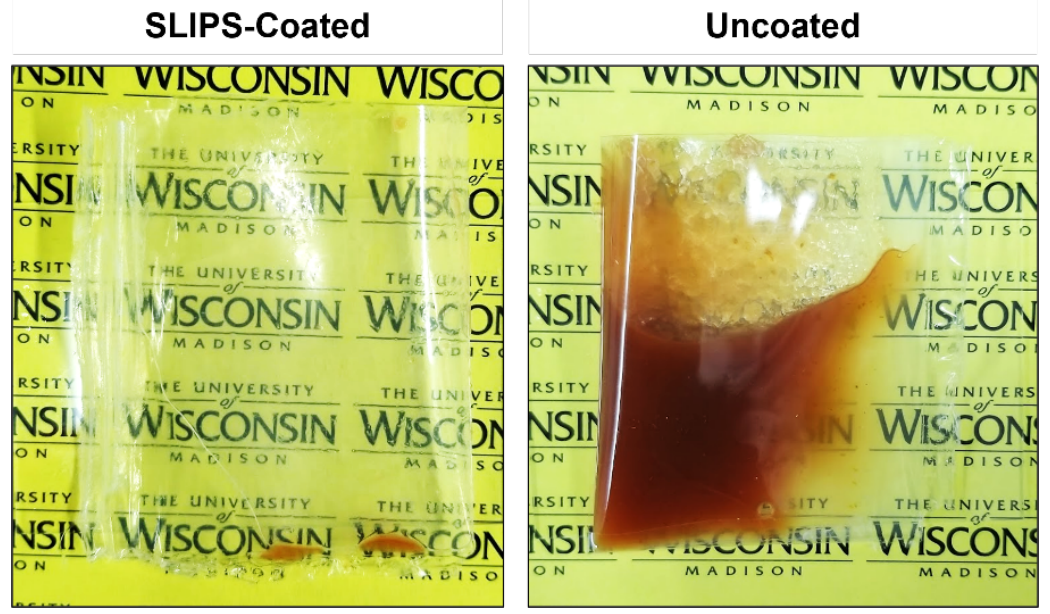

Figure S10. Images showing SLIPS-coated and uncoated flexible pouches after exposure to ketchup. The pouches were filled with tomato ketchup and then tilted and shaken to dispense the ketchup. While most of the ketchup slid out of the SLIPS-coated pouch, the uncoated pouch was heavily fouled by the remaining ketchup in the bag even after vigorous shaking (see Video S2).

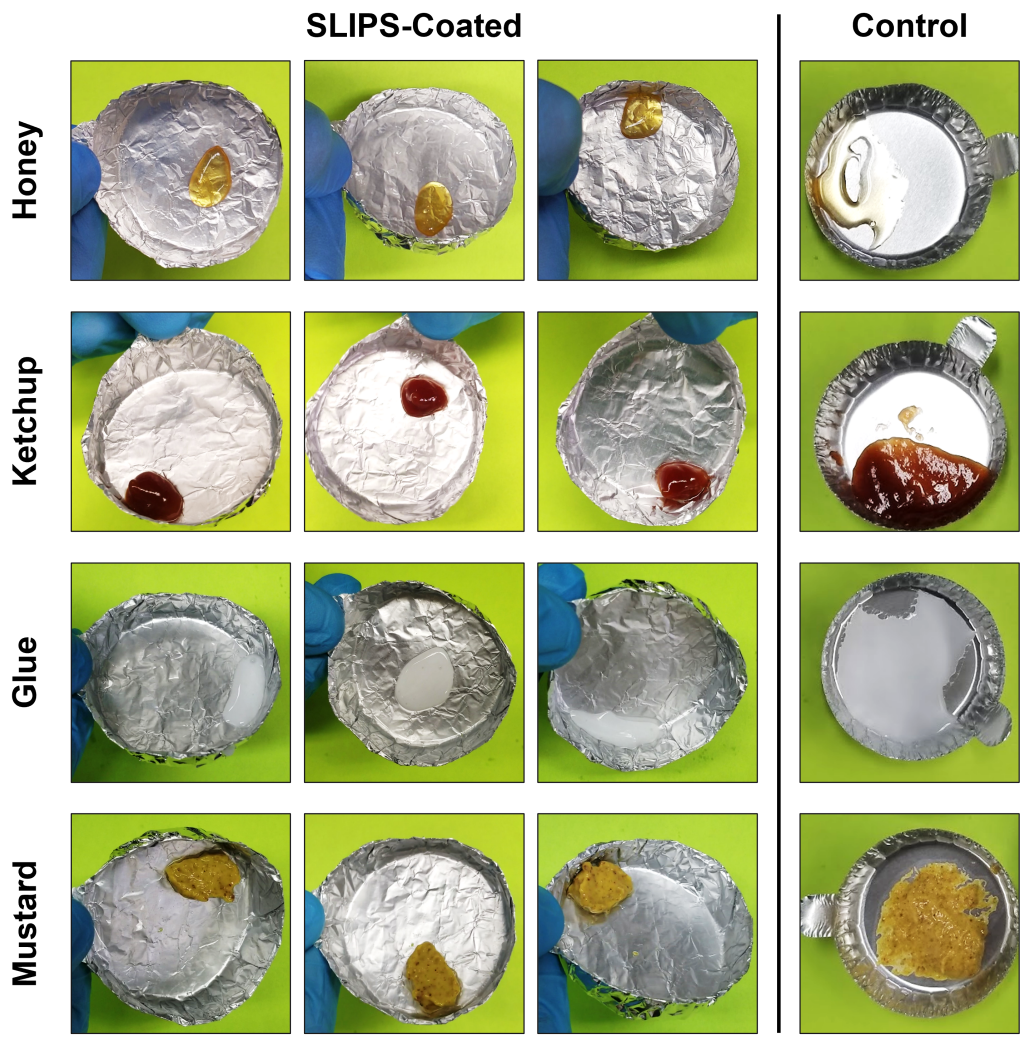

Figure S11. Series of images showing samples of different commercially relevant liquids (honey, ketchup, glue, and mustard) sliding inside SLIPS-coated aluminum foil containers fashioned from strips of SLIPS-coated foil (also see Video S3). All of the fluids heavily fouled the surfaces of uncoated containers (show in the images in the column on the right). Images showing sliding of honey on SLIPS-coated aluminum foil dish are reproduced from Figure 7. 

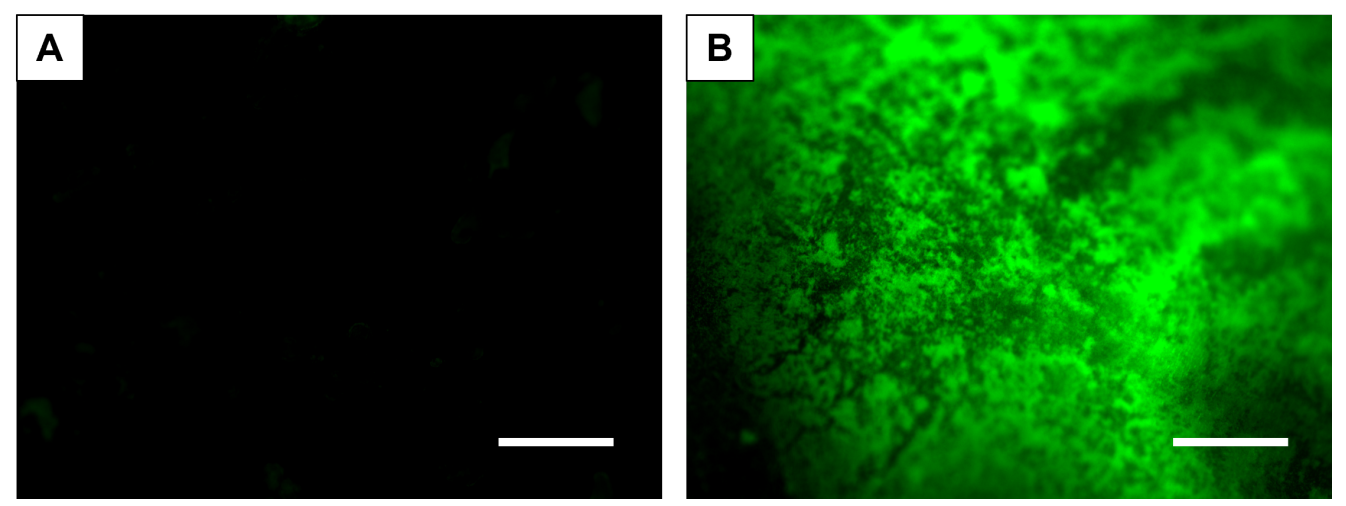

Figure S12. Fluorescence microscopy images of SLIPS-coated (A) and uncoated (B) aluminum foils after incubation with $S$. aureus for 24 hours; samples were treated with the fluorescent stain SYTO-9 prior to imaging (see Methods). Scale bars $=400 \mu \mathrm{m}$. The fluorescence (false-colored green) observed on the uncoated substrate in (B) indicates fouling by bacteria; the SLIPS-coated substrate shown in (A) remained free of biofouling.

\section{$\underline{\text { Supporting Videos }}$}

Video S1. A roll-to-roll spraying process showing a polyethylene substrate being pulled from a feed roll (right) to a collection roll attached to a motor (left). The pull rate shown here is $\sim 0.6$ $\mathrm{cm} . \mathrm{sec}^{-1}$. The substrate passes under two stationary spraying nozzles (a separate nozzle for both PEI and PVDMA solutions). This video is a companion to the still image shown in Figure 6A.

Video S2. A SLIPS-coated polyethylene pouch fashioned from a continuously manufactured spray-coated $10 \times 20 \mathrm{~cm}$ substrate. The pouch is shown with ketchup added and is inverted, allowing the ketchup to slide out. An uncoated polyethylene bag is also shown filled with ketchup and then inverted. This video is a companion to the still images shown in Figure 7A-C and Figure S10.

Video S3. Droplets of various liquids (honey, ketchup, glue, and mustard) sliding inside a SLIPS-coated aluminum foil dish. For all other fluids except honey, the video playback speed has been increased to $2 \mathrm{x}$. Video demonstrating the sliding of honey inside the aluminum foil dish is played at $4 \mathrm{x}$ the original speed. This video is a companion to the still image shown in Figures 7D-F and S11.

Video S4. A SLIPS sticker affixed (in the center) to a glass slide. Ketchup is added across the top of the glass slide. The ketchup slides easily on the SLIPS sticker, and the surface of the sticker remains unfouled, whereas the surface of the glass slide outside the sticker is heavily fouled. This video is a companion to the still image shown in Figure 7G-I. 